\title{
Endoscopic endonasal surgical management of chondrosarcomas with cerebellopontine angle extension
}

\author{
Paulo M. Mesquita Filho, M.D., ${ }^{1}$ Leo F. S. Ditzel Filho, M.D., ${ }^{1}$ \\ Daniel M. Prevedello, M.D., ${ }^{1,2}$ Cristian A. N. Martinez, M.D., ${ }^{1}$ \\ Mariano E. Fiore, M.D., ${ }^{1}$ Ricardo L. L. Dolci, M.D., ${ }^{2}$ Bradley A. Otto, M.D., ${ }^{1,2}$ \\ and Ricardo L. Carrau, M.D. ${ }^{1,2}$
}

Departments of ${ }^{I}$ Neurosurgery and ${ }^{2}$ Otolaryngology-Head \& Neck Surgery, Wexner Medical Center at The Ohio State University, Columbus, Ohio

\begin{abstract}
Object. Skull base chondrosarcomas are slow-growing, locally invasive tumors that arise from the petroclival synchondrosis. These characteristics allow them to erode the clivus and petrous bone and slowly compress the contents of the posterior fossa progressively until the patient becomes symptomatic, typically from cranial neuropathies. Given the site of their genesis, surrounded by the petrous apex and the clival recess, these tumors can project to the middle fossa, cervical area, and posteriorly, toward the cerebellopontine angle (CPA). Expanded endoscopic endonasal approaches are versatile techniques that grant access to the petroclival synchondrosis, the core of these lesions. The ability to access multiple compartments, remove infiltrated bone, and achieve tumor resection without the need for neural retraction makes these techniques particularly appealing in the management of these complex lesions.

Methods. Analysis of the authors' database yielded 19 cases of skull base chondrosarcomas; among these were 5 cases with predominant CPA involvement. The electronic medical records of the 5 patients were retrospectively reviewed for age, sex, presentation, pre- and postoperative imaging, surgical technique, pathology, and follow-up. These cases were used to illustrate the surgical nuances involved in the endonasal resection of CPA chondrosarcomas.

Results. The male/female ratio was 1:4, and the patients' mean age was $55.2 \pm 11.2$ years. All cases involved petrous bone and apex, with variable extensions to the posterior fossa and parapharyngeal space. The main clinical scenario was cranial nerve $(\mathrm{CN})$ palsy, evidenced by diplopia (20\%), ptosis $(20 \%)$, CN VI palsy (20\%), dysphagia $(40 \%)$, impaired phonation $(40 \%)$, hearing loss $(20 \%)$, tinnitus $(20 \%)$, and vertigo/dizziness $(40 \%)$. Gross-total resection of the CPA component of the tumor was achieved in 4 cases $(80 \%)$; near-total resection of the CPA component was performed in 1 case (20\%). Two patients (40\%) harbored high-grade chondrosarcomas. No patient experienced worsening neurological symptoms postoperatively. In 2 cases $(40 \%)$, the symptoms were completely normalized after surgery.

Conclusions. Expanded endoscopic endonasal approaches appear to be safe and effective in the resection of select skull base chondrosarcomas; those with predominant CPA involvement seem particularly amenable to resection through this technique. Further studies with larger cohorts are necessary to test these preliminary impressions and to compare their effectiveness with the results obtained with open approaches.
\end{abstract}

(http://thejns.org/doi/abs/10.3171/2014.7.FOCUS14349)

$\begin{array}{lllll}\text { KEY WORDS } & \bullet \quad \text { chondrosarcoma } & \bullet & \text { cerebellopontine angle } \quad \bullet \quad \text { endoscopic } & \bullet \\ \text { endonasal } & \bullet & \text { skull base }\end{array}$

$\mathrm{C}$ HONDROSARCOMAS of the skull base are malignant tumors with an indolent growth and low metastatic chondrosis, these lesions usually compromise the brainstem, cranial nerves, and important vascular structures. Due to these features, patients harboring these lesions often have significant neurological deficits and tumors with large dimensions when first diagnosed. The critical

Abbreviations used in this paper: $\mathrm{CN}=$ cranial nerve; $\mathrm{CPA}=$ cerebellopontine angle; EEA = endoscopic endonasal approach; ICA = internal carotid artery; IMRT = intensity-modulated radiation therapy. neurovascular structures that surround the tumor render their surgical treatment complex, extensive, and hazardous, often resulting in significant morbidity. This is best exemplified by the subset of patients in whom the lesion progresses toward the posterior cranial fossa, displacing the cranial nerves and compressing the brainstem. These tumors commonly present with a main component located primarily on the cerebellopontine angle (CPA) region; their transcranial surgical management is therefore naturally based on the retrosigmoid and variations of the transpetrous approaches. Conversely, expanded endoscopic endonasal approaches (EEAs) have recently become a tool of interest in skull base surgery, offering a direct anterior approach to these lesions, which allows 
adequate resection while minimizing surgical morbidity. Likewise, the ability to access multiple compartments, remove infiltrated bone, and achieve tumor resection without the need for neural retraction makes EEAs particularly appealing in the management of these complex lesions.

We advocate the use of EEAs as the initial surgical corridor in all cases of petroclival chondrosarcomas, since the origin of these lesions is the petroclival synchondrosis. EEAs provide direct access to virtually the entire ventral skull base; because chondrosarcomas typically arise and grow from the petroclival region with varying patterns of progression, they tend to displace neurovascular elements laterally, superiorly, and posteriorly. Hence, an endonasal corridor will permit the surgeon to access the tumor's main core and "follow" it into different compartments of the skull base while avoiding the need for any cerebral retraction or cranial nerve manipulation. Furthermore, this approach allows extensive drilling of the ventral bone structures of the skull base, particularly the clivus, sphenoid, and petrous portions of the temporal bone, which are often infiltrated and eroded by these tumors.

Nonetheless, one must be aware that the term "endoscopic endonasal approach" is broad and refers to a multitude of approaches that use the endonasal corridor to reach a ventrally located target. These approaches invariably rely on a transsphenoidal window, which is coupled with different anatomical modules according to tumor location. Lesions primarily located in the upper petroclival region with cavernous sinus or middle cranial fossa involvement may be accessed through a transsphenoidal approach with removal of the sphenoid and temporal bony encasement over these regions. Lesions in the middle third of the clivus may be accessed with a transsphenoidal approach and associated clivectomy and anterior petrosectomy. Lesions in the lower clivus with infratemporal fossa extension require a transpterygoid approach. In summary, each approach is tailored to the specific location of the tumor in the individual patient.

This study, however, focuses on skull base chondrosarcomas with CPA involvement-i.e., tumors that, despite secondary components in other skull base regions, were primarily located in the CPA. This feature was determined solely on the basis of preoperative scans. In some cases, when the meningeal layer of dura had minimal or no violation and was posteriorly displaced, the tumor did not reach the arachnoid space. Because of their precise location these lesions were still classified as CPA tumors for didactic reasons, but we acknowledge that in these cases the CPA classification might be a misnomer. Nonetheless, the current series describes the role of EEAs in the surgical management of 5 chondrosarcomas with CPA involvement, the need for combining these with transcranial approaches, and the results yielded by this strategy.

\section{Methods}

We retrospectively reviewed all cases of chondrosarcomas with predominant involvement of the posterior fossa and CPA that were surgically treated at the Ohio State University between July 2010 and January 2014.

Patients' charts, imaging studies, and pathological records were examined. Data on the location of the tumor, degree of resection, and follow-up imaging status were taken from imaging studies. Preoperative and postoperative clinical condition as well as follow-up clinical status were taken from the charts. Histological type and grades were taken from the biopsy samples.

\section{Patient Selection and Surgical Technique}

At our center, the parameters that dictate approach selection for all skull base tumors rely on choosing the single approach or combination of approaches that may allow the maximal, safe tumor resection while avoiding crossing the trajectory of the main cranial nerves displaced by the lesion. Chondrosarcomas of the skull base most commonly present as off-midline tumors centered on the petroclival synchondrosis, displacing relevant neurovascular structures laterally, posteriorly, superiorly, and/or inferiorly. Given these scenarios, EEAs are usually elected as the approach of choice for initial management, given that they provide a ventral direct corridor to the target without the need to retract or displace neurovascular structures, which are typically located on the periphery of the tumor. Hence, virtually all skull base chondrosarcomas are approached endonasally, at least initially, at our center.

The surgical technique of endonasal transpterygoid approaches to the petrous apex and petroclival regions has been previously described. ${ }^{8}$ In summary, a wide transsphenoidal approach is performed under fully endoscopic visualization after a pedicled nasoseptal flap has been raised on the contralateral side. A wide antrostomy permits visualization of the posterior wall of the maxillary sinus; removal of this wall grants access to the contents of the pterygopalatine fossa. Occlusion of the posterior nasal and sphenopalatine arteries allows for lateral displacement of these contents, thus permitting identification of the vidian nerve and its canal. This canal is followed posteriorly until its junction with the foramen lacerum. The floor of the sphenoid sinus is drilled flush with the clivus; the clivus itself is drilled down until the dura of the posterior fossa is visualized. Skeletonization of the paraclival internal carotid artery (ICA) permits its lateral displacement, thus granting the surgeon access to the entire petroclival synchondrosis. At this point, tumor is usually visible surrounding the foramen lacerum; since these lesions are usually soft and yield to direct aspiration, one can enter the tumor's core and "follow" it until all secondary components have been removed. Typically, at the end of the resection, the surgeon encounters the posterior cranial fossa dura "dented" by the tumor's posterior progression. Often, these tumors destroy the periosteal dural layer and preserve the meningeal layer, which still separates the lesion from the subarachnoid space. There are cases in which the meningeal layer is eroded and the tumor assumes a position in the subarachnoid space. This component of the tumor is followed intradurally under direct visualization if necessary. Infratemporal fossa extensions require an additional transpterygoid approach, in 


\section{Chondrosarcomas with CPA extension}

which the medial and, sometimes, the lateral pterygoid plates (previously exposed by the lateral displacement of the pterygopalatine fossa contents) are drilled down. Removal of the ipsilateral torus tubarius enhances this exposure. Finally, when resection is completed, the contralateral nasoseptal flap is applied covering mainly the exposed ICA and the posterior cranial fossa dura.

\section{Results}

Retrospective review of our database yielded 5 cases; the pertinent tumor characteristics are presented in Table 1 , and the patients' clinical and demographic characteristics are summarized in Table 2.

Four patients were women and 1 patient was a man. The mean age $( \pm \mathrm{SD})$ was $55.2 \pm 11.2$ years (range $40-66$ years). The main clinical scenario was cranial nerve $(\mathrm{CN})$ palsy, evidenced by diplopia (20\%), ptosis (20\%), CN VI palsy (20\%), dysphagia (40\%), impaired phonation (40\%), hearing loss (20\%), tinnitus (20\%), and vertigo/dizziness $(40 \%)$. One patient $(20 \%)$ presented with headache, nausea, and vomiting, which may have been related to elevated intracranial pressure.

All cases involved the petrous bone and apex, with variable extensions to the clivus (80\%), CPA (100\%), jugular foramen (60\%), internal acoustic canal (20\%), hypoglossal canal (20\%), middle cranial fossa (20\%), and parapharyngeal space (20\%), and surrounding the ICA (40\%).

Gross-total resection of the CPA component of the tumor was achieved in 4 patients $(80 \%)$. The patient with a near-total resection of the CPA component of the tumor underwent a retromastoid craniectomy to complete the resection, as well as a transoral approach to remove a small remnant in the parapharyngeal space. This strategy resulted in a gross-total resection of this specific lesion. EEA alone was able to provide gross-total resection of the entire tumor in 2 patients $(40 \%)$, and near-total resection (> 95\%) was performed in 3 cases $(60 \%)$. Two patients $(40 \%)$ harbored high-grade chondrosarcomas, and 2 patients $(40 \%)$ had low-grade lesions. One patient (20\%) had an intermediate (Grade II) lesion. All patients received some type of radiation; 4 patients $(80 \%)$ received proton beam therapy.

The mean follow-up period was $14.8 \pm 12.1$ months (range 3-33 months). No patient experienced worsening of neurological symptoms after the procedure. Two patients (40\%) experienced complete resolution of their symptoms. Three patients $(60 \%)$ continued to have mild symptoms, which were already present preoperatively. None of the patients died. There were no postoperative cerebrospinal fluid leaks, and lumbar drains were not used in any of the cases.

\section{Case Reports}

\section{Case 1}

A 47-year-old woman presented for consultation with diplopia, headaches, nausea, and vomiting as well as right ptosis. A brain MRI study was performed, revealing a T2 hyperintense, T1-enhancing mass at the right petrous 
TABLE 2: Comparison of pre- and postoperative clinical condition in 5 cases*

\begin{tabular}{ccllr}
\hline & & \multicolumn{2}{c}{ Condition } & \\
\cline { 3 - 4 } Case No. & Age $(\mathrm{yrs}), \mathrm{Sex}$ & \multicolumn{1}{c}{ Preop } & \multicolumn{1}{c}{ Postop } & Follow-Up \\
\hline 1 & $47, \mathrm{~F}$ & diplopia, headaches, N/V, rt ptosis & no neurol deficits & 3 mos \\
2 & $62, \mathrm{M}$ & deteriorated phonation, dysphagia & no neurol deficits & 20 mos \\
3 & $40, \mathrm{~F}$ & rt CN VI palsy & rt CN VI palsy & 33 mos \\
4 & $61, \mathrm{~F}$ & hearing loss, dizziness, ataxia & hearing loss & 10 mos \\
5 & $66, \mathrm{~F}$ & dysphagia, phonation impairment, vertigo, tinnitus & tinnitus & 10 mos \\
\hline
\end{tabular}

* neurol = neurological; $\mathrm{N} / \mathrm{V}$ = nausea and vomiting.

apex, with involvement of the midclivus. Due to this finding, the patient underwent, at a different neurosurgical department, a transsphenoidal biopsy of the disease in the sphenoid sinus, as well as a combined transpetrous subtemporal craniotomy for additional debulking. Following surgery, the patient had complete relief of symptoms, and the pathological analysis resulted in a diagnosis of Grade II chondrosarcoma. A postoperative MRI study showed a partial resection of the tumor (Fig. 1a and b). The patient was referred for proton beam therapy. This treatment was not advised, however, due to possible effects of radiation near to critical anatomical structures. The patient was then referred to our neurosurgical department to discuss the possibility of further tumor resection. She eventually underwent an EEA, with near-total resection (>95\%)
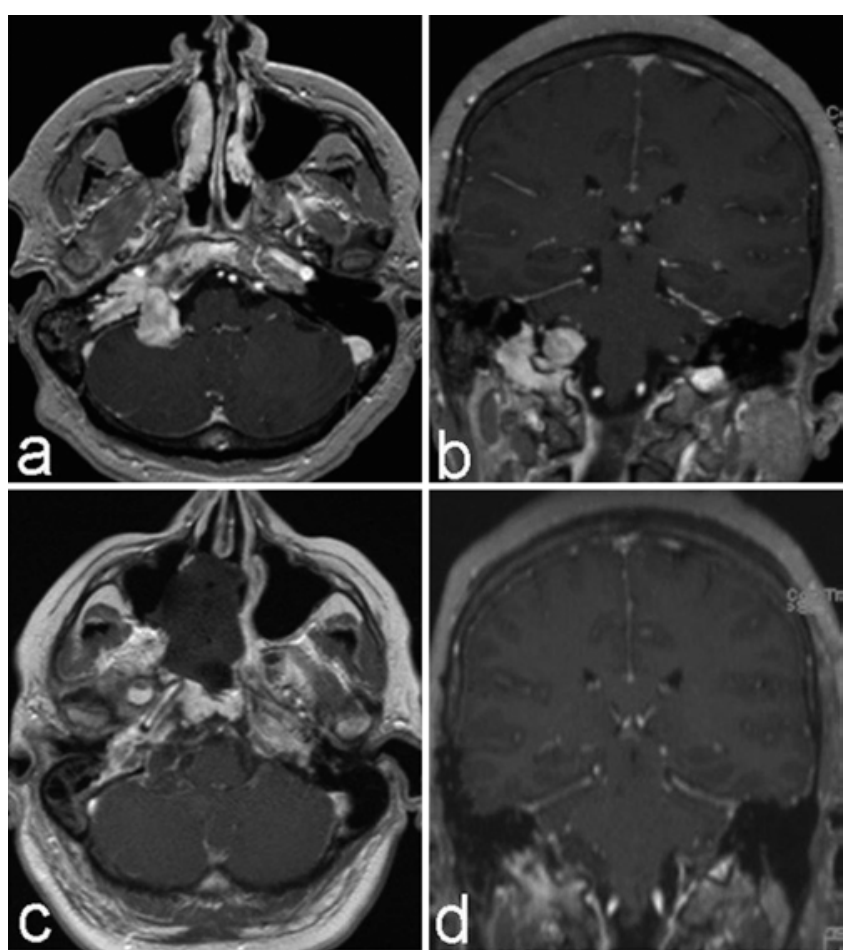

FIG. 1. Case 1. Gd-enhanced T1-weighted MR images obtained after the initial debulking procedure ( $a$ and $b)$ and after the endoscopic endonasal approach and subtotal resection (c and d). a: Axial image revealing tumor involving the right petrous bone and clivus with extension to the CPA and the right cerebellum. b: Coronal image highlighting the CPA extension. $\quad$ c and d: Postoperative axial (c) and coronal (d) images showing a small residual lesion in the right mastoid. of the lesion overall and complete resection of the CPA component (Fig. 1c and d). There was a small amount of residual tumor in the right mastoid, as well as in the right superior orbital fissure. We proposed another procedure for resection, but the patient preferred to undergo adjuvant proton beam therapy. As of this writing, 1 month after the endoscopic endonasal procedure, the patient remains clinically intact.

\section{Case 2}

A 62-year-old man presented with a previous history of surgery through a left retromastoid approach (in 1991) for resection of a chondrosarcoma. At the time, the resection was followed by 2 Gamma Knife procedures. Recently, the patient had experienced deterioration of phonation and developed dysphagia. Reinvestigation demonstrated a large tumor occupying the clivus and petrous bone on the left side, with progression through the jugular vein into the neck region, as well as intradural progression to the posterior fossa, occupying an intradural position in the left portion of the cerebellum (Fig. 2a-c). An EEA was proposed for resection of the recurrent tumor. The sample obtained during surgery was compatible with a Grade II/III chondrosarcoma. After the procedure, the patient was treated with proton beam therapy. Follow-up images revealed total resection of the tumor, including the components inside the CPA and around the jugular vein (Fig. 2d-f). As of this writing, 20 months after the procedure, there has been no evidence of recurrence and the patient is clinically intact.

\section{Case 3}

A 40-year-old woman presented with an 8-month history of right $\mathrm{CN}$ VI palsy. Investigation with $\mathrm{CT}$ and MRI showed the presence of a lesion in the clivus with posterior fossa involvement and brainstem compression (Fig. 3a and b). The images also revealed a very large tumor component in the right middle fossa and complete destruction of the petrous apex. The right temporal lobe was displaced and another component of the tumor was descending around the internal carotid artery on the right side, filling the area with tumor all the way around the right jugular foramen and hypoglossal canal. The patient underwent an EEA for resection of the lesion; surgery was uneventful. Postoperative MRI showed a complete resection of the CPA component of the tumor, with residual tumor in the right middle fossa, above the petrous bone 


\section{Chondrosarcomas with CPA extension}
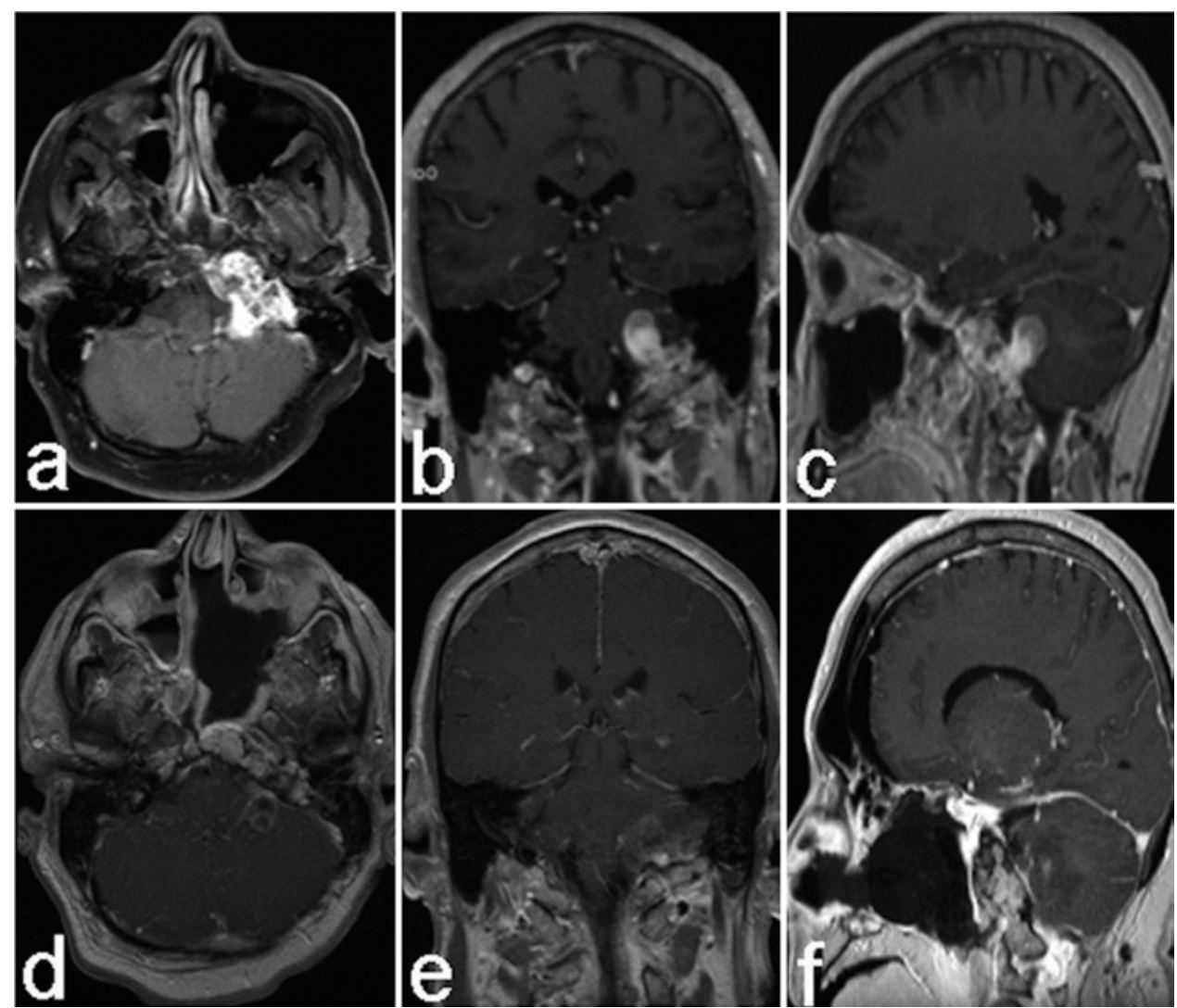

FIG. 2. Case 2. Preoperative and postoperative Gd-enhanced T1-weighted MR images. a-c: Preoperative axial, coronal, and sagittal images revealing tumor involving the left petrous bone and clivus, with extension to the CPA. d-f: Postoperative axial, coronal, and sagittal Gd-enhanced T1-weighted MR images showing gross-total resection.

and also in the right condyle and jugular foramen (Fig. 3c and d). Due to these findings, a second-stage endoscopic endonasal surgery was performed. The results of pathological examination were compatible with the diagnosis of Grade I chondrosarcoma. Postoperative images obtained after this second endoscopic endonasal procedure showed a near-total resection of the tumor overall with minimal residual lesion in the right occipital condyle. The patient was referred for radiation therapy (6560 cGy of 6 MV photons in 32 fractions to the right skull base and right middle cranial fossa with an intensity-modulated radiation therapy [IMRT] technique). Thirty-three months after the endoscopic endonasal procedures, the patient's condition remains stable with a mild CN VI palsy, which was not as severe as it had been before surgery.

\section{Case 4}

A 61-year-old woman presented with hearing loss, dizziness, and ataxia. MRI investigation evidenced a mass involving the left petrous bone, surrounding the ICA and extending to the CPA, with important compression of the brainstem (Fig. 4a and b). An EEA was proposed (Fig. 5), and a near-total resection $(>95 \%)$ was achieved. There was a small amount of residual tumor in the CPA as well as in the cervical parapharyngeal space (Fig. $4 \mathrm{c}$ and d). A 2 -procedure second stage was performed. The lesion on the CPA was approached through a retrosigmoid craniotomy, while the cervical component was reached trans- orally during the same anesthesia session. The findings of the pathological examination were compatible with a Grade II/III chondrosarcoma. The patient was referred for proton beam therapy after the second procedure, and postoperative MRI confirmed total resection (Fig. 4e and f). Ten months after the endoscopic endonasal surgery, the patient has a persistent hearing loss but no other neurological deficits.

\section{Case 5}

A 66-year-old woman presented with a history of progressive dysphagia, impaired phonation, vertigo, and tinnitus. The MRI investigation revealed a large enhancing mass in the right petrous bone and clivus, with a descending path to the level of hypoglossal canal (Fig. $6 \mathrm{a}$ and $\mathrm{b}$ ). There was also a posterior component compressing the brainstem and the cerebellum. An EEA was performed, and a gross-total resection was accomplished, including the CPA component of the tumor (Fig. 6c and d). The pathological examination findings were compatible with a low-grade chondrosarcoma. After the surgery, the patient was referred for proton beam therapy. As of the most recent follow-up evaluation, 10 months after the procedure (Fig. 6e and f), she still had mild tinnitus, but her other deficits had resolved.

\section{Discussion}

Chondrosarcomas are rare slow-growing malig- 

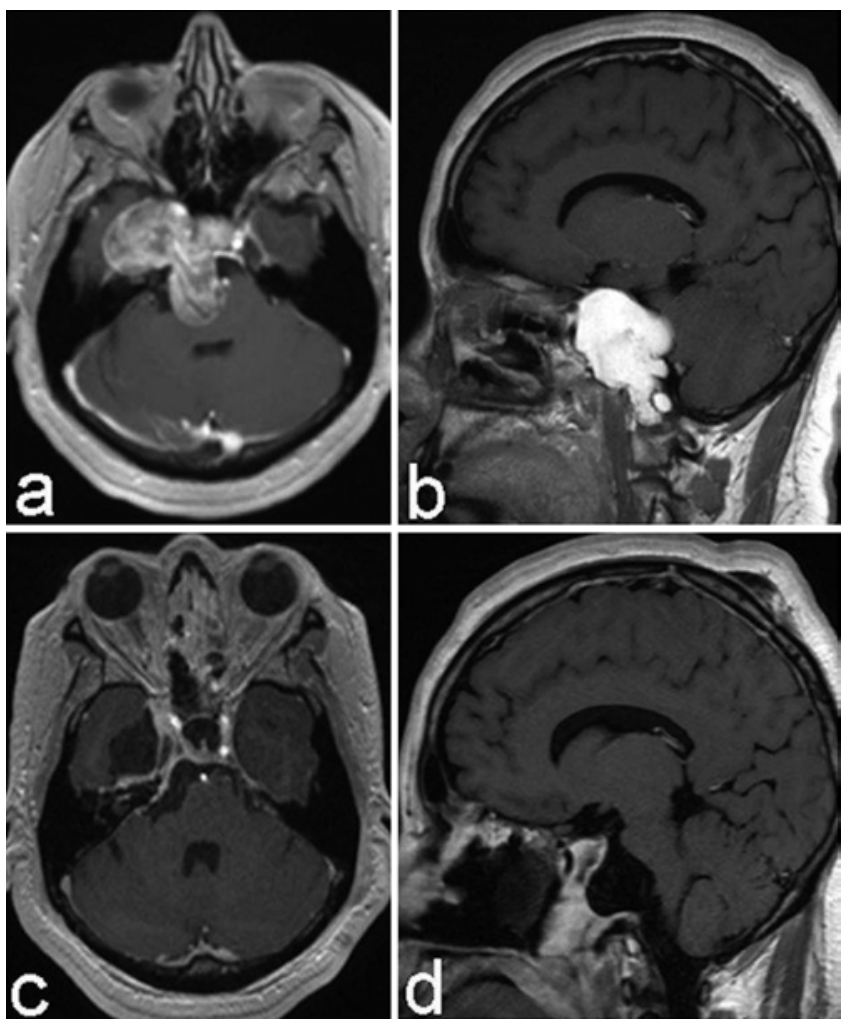

FIG. 3. Case 3. Gd-enhanced T1-weighted MR images. a: Preoperative axial image revealing an extensive mass involving the right petrous bone and clivus, the middle fossa, Meckel's cave, and the sellar region with extension to the CPA. b: Preoperative sagittal image showing extensive mass in the clivus and petrous bone. $\mathbf{c}$ and $\mathbf{d}$ : Postoperative axial (c) and sagittal (d) images showing near-total resection, with a remnant posterolateral to the ICA and in the right occipital condyle.

nant bone tumors composed of chondroid-origin cells throughout the axial and appendicular skeleton. ${ }^{3,4,15}$ They often arise from the lateral aspects of the skull base that house cartilage, including the temporooccipital synchondrosis, the sphenoocciput, and the sphenoethmoid complex. ${ }^{7}$ The structure most often involved in cases of skull base chondrosarcomas is the clivus (32\%), followed by other synchondroses. ${ }^{6}$ These tumors can also involve the cavernous sinus, petrous bone, and sphenoid bone. ${ }^{7}$ In the skull base, $64 \%$ of chondrosarcomas arise in the middle fossa, $14 \%$ involve both the middle and the posterior fossa, $14 \%$ occur in the anterior fossa, and $7 \%$ originate in the posterior fossa. ${ }^{3,4}$ In the present series, tumor components extended to the CPA in 5 (26.3\%) of the 19 cases of skull base chondrosarcomas in our database.

Chondrosarcomas are classified into 4 histological groups: conventional (majority), mesenchymal (< 10\%), clear cell, and dedifferentiated. The major histological differential diagnosis is chondroid chordoma. Since chordoma is a malignant tumor derived from the primitive notochord, it typically arises at the clivus, in the midline, and it should also have recognizable epithelial differentiation characteristics. The absence of definitive notochordal elements is indicative of chondrosarcoma. ${ }^{4}$ Almefty et al. highlighted the fact that this differentiation is important
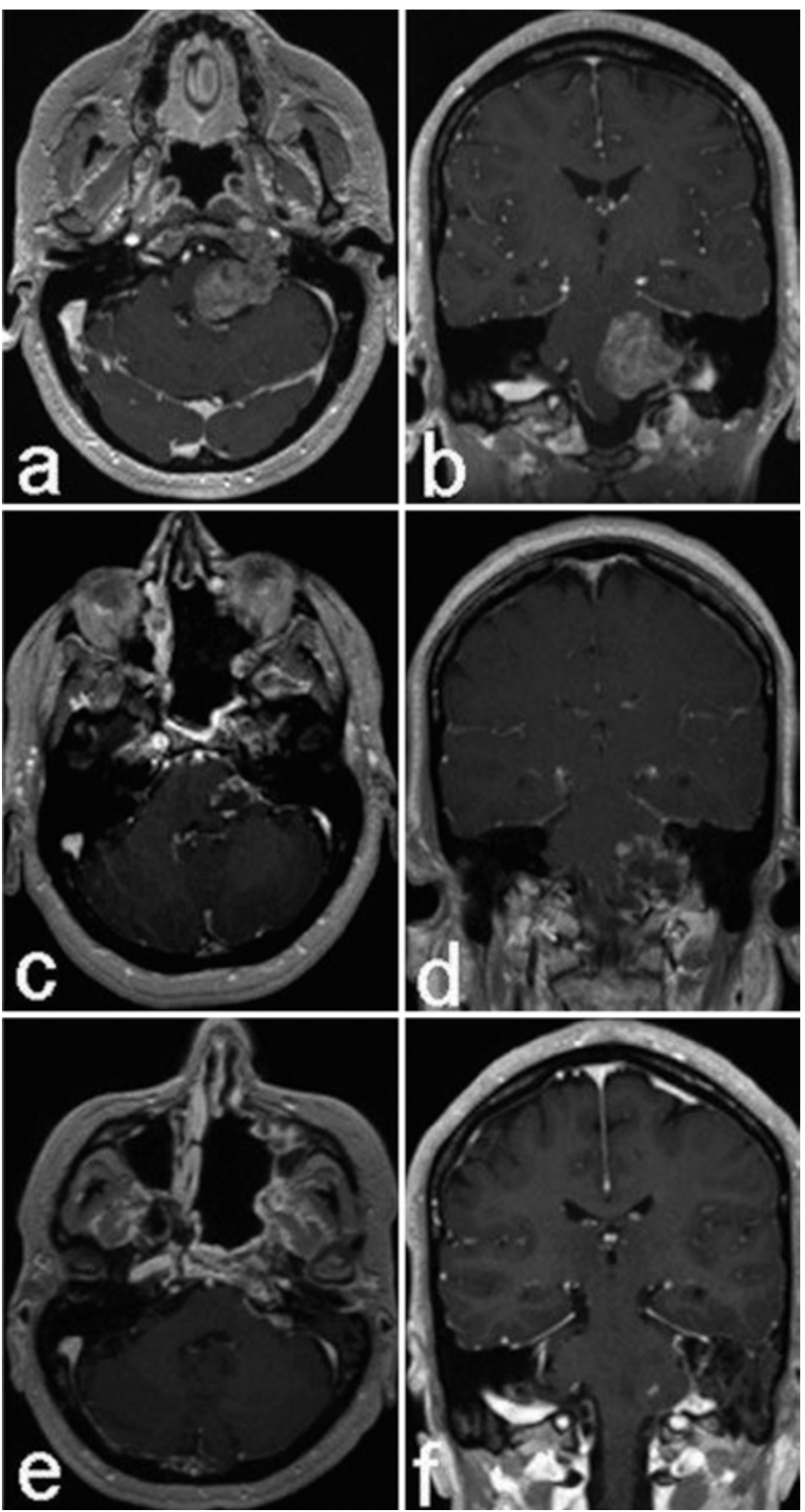

FIG. 4. Case 4. Preoperative and postoperative Gd-enhanced T1weighted MR images. a: Preoperative axial image revealing tumor involving the left petrous bone and ICA, with extension to the CPA and compression of the brainstem. b: Preoperative coronal image showing tumor displacing the brainstem. $\quad \mathbf{c}$ and d: Postoperative axial (c) and coronal (d) images showing a small remnant in the CPA. e and f: Axial (e) and coronal (f) images from the most recent follow-up evaluation (10 months after the second procedure) showing total resection of the CPA lesion.

even for patient outcome. Chordomas demonstrated aggressive clinical course and poor outcome, while patients with chondrosarcoma have a significantly better outcome with regard to survival and recurrence-free survival without high-dose radiotherapy. ${ }^{1,11}$

The symptomatology is related to the location of these lesions. The most common symptoms are headache and diplopia. Other cranial nerve palsies, manifested as hoarseness, dysphagia, facial dysesthesia, and hearing loss, and gait disturbances, may also occur. ${ }^{3,4}$ Headache 


\section{Chondrosarcomas with CPA extension}
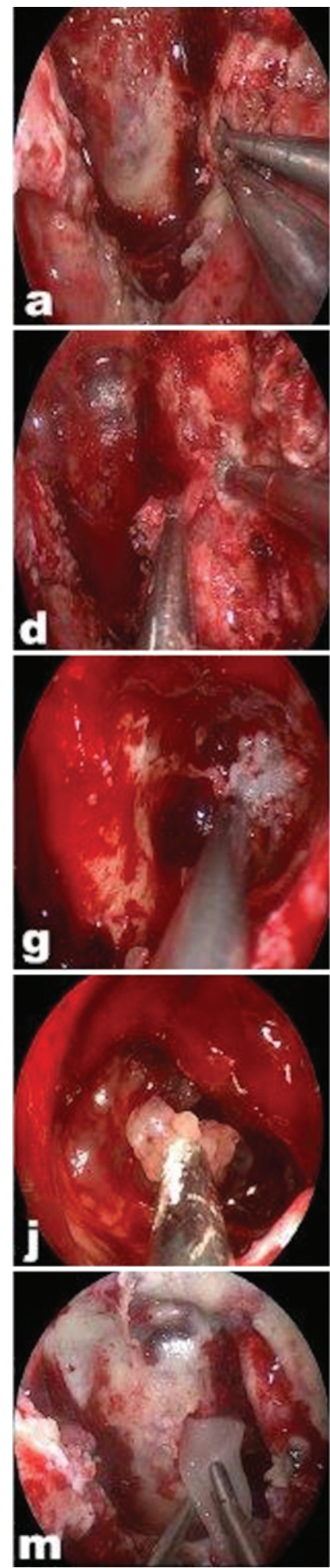
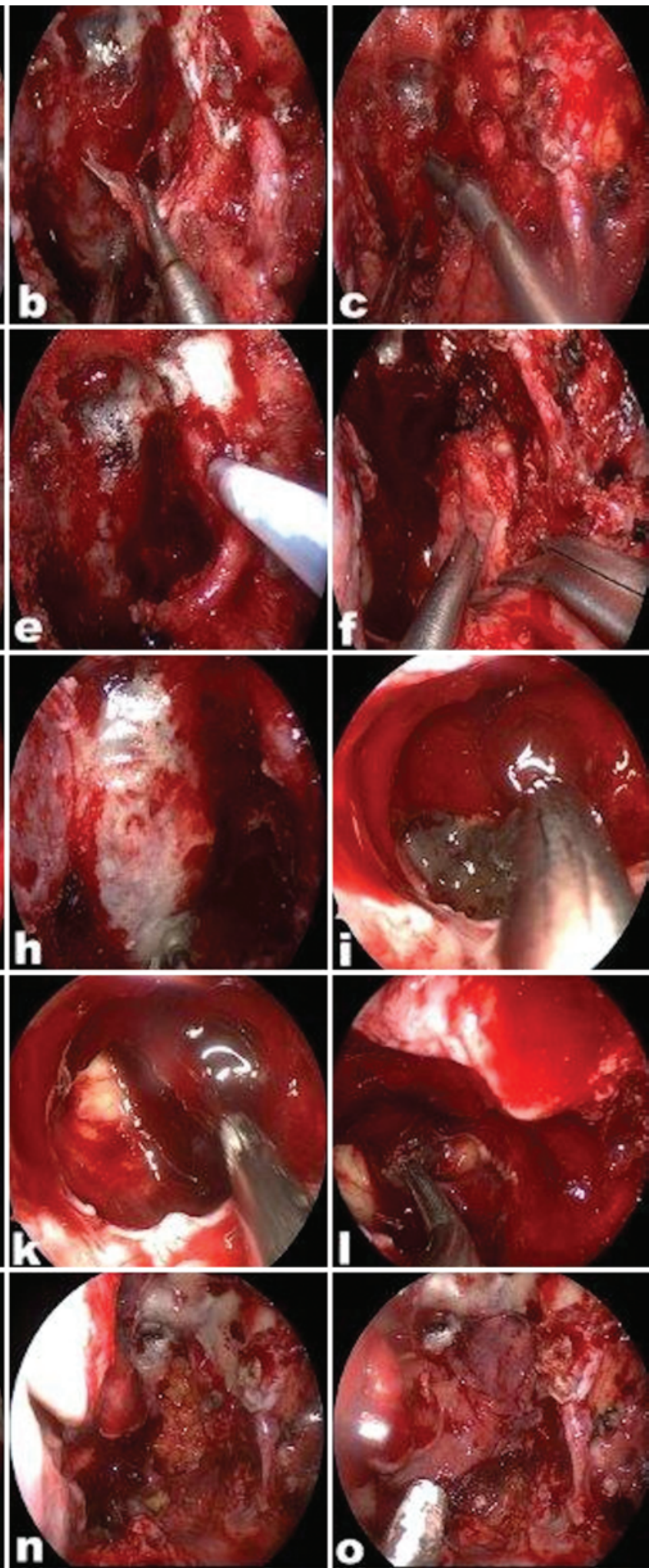

FIG. 5. Case 4. Intraoperative photographs. a: Initial drilling and detaching of the medial pterygoid plate. b: Exposure of tumor lateral to the bone surface. c: Biopsy sample being taken. d: Further drilling inferior to the vidian nerve. e: Use of the Doppler probe to identify the ICA. f: After identification and exposure of the paraclival and petrous ICA, more exposure was necessary to access the tumor behind the artery, and the eustachian tube was incised. $\mathbf{g}$ : Removal of tumor behind and lateral to the ICA. h: Drilling in the inferomedial aspect of the clivus. i: Tumor in the inferomedial aspect of the clivus. j: Further debulking of the lesion. k: Exposure of the brainstem after debulking of the lesion. I: Final aspect of the operative site, before reconstruction. m-o: Multilayer reconstruction with collagen matrix, fat, and finally, the pedicled nasoseptal flap. 

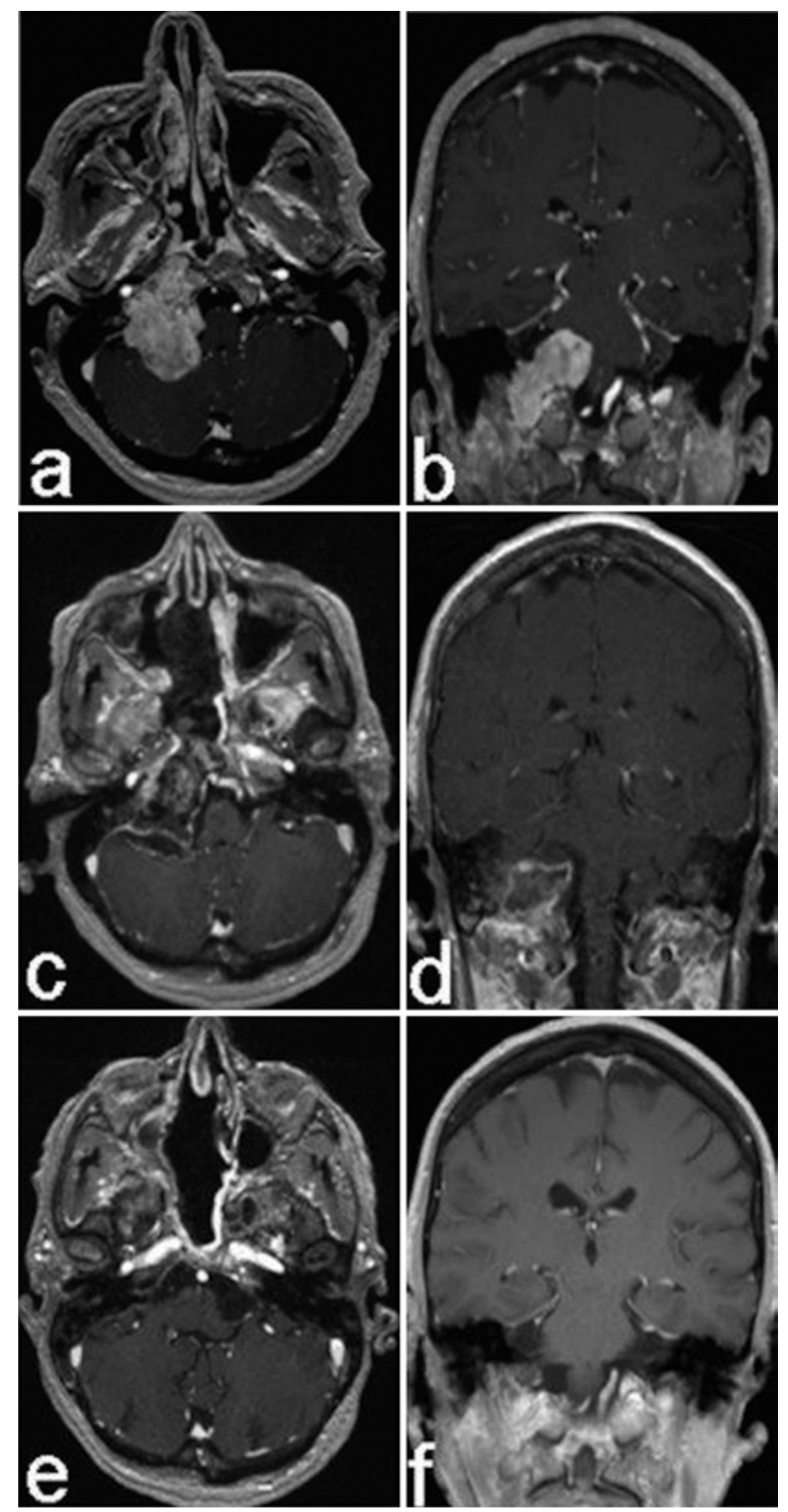

FIG. 6. Case 5. Preoperative and postoperative Gd-enhanced T1weighted MR images. a: Preoperative axial image revealing tumor involving the right petrous bone, with extension to the CPA and compression of the brainstem. b: Preoperative coronal image showing tumor displacing the brainstem, with a downward extension. $\mathbf{c}$ and d: Immediate postoperative axial (c) and coronal (d) images demonstrating gross-total resection. e and f: Axial (e) and coronal (f) images from the most recent follow-up evaluation (10 months after surgery) showing no evidence of recurrence.

may result from irritation of the skull base dura mater or intracranial hypertension. Diplopia is related to involvement of the clivus, with CN VI compression at the entrance of Dorello's canal even with minimal mass effect, and it is present in nearly $50 \%$ of the cases. ${ }^{6}$ One group reported on 8 patients with a predominance of petrous apex tumors extending into the CPA and described all 8 as presenting with dysfunction of the lower cranial nerves. ${ }^{3,4}$ The presence of specific cranial nerve deficits is important in the selection of the surgical approach. ${ }^{3}$

Imaging studies have an important role in defining diagnosis and planning the surgical approach. ${ }^{4} \mathrm{CT}$ scan demonstrates bony destruction of the skull base lateral to the midline; the typical appearance is a destructive lesion with scalloped erosive borders. MRI better delineates soft tissue details and helps to evaluate tumor involvement of neural and vascular structures. The tumor has lowto-intermediate signal intensity on T1-weighted images and high signal intensity on T2-weighted images. The enhancement is usually marked and signal heterogeneity is frequently observed in postcontrast images because of matrix mineralization and prominent fibrocartilaginous elements within the tumor., ${ }^{412}$ Angiographic studies also play an important role, especially in surgical planning, since major vessels can be encased by the tumor.

Skull-base chondrosarcomas are indolent but locally invasive malignancies in locations that are often difficult to treat, and they have a propensity for recurrence if inadequately treated. ${ }^{3,4}$ Although it is agreed that surgery is the initial treatment of choice, there is controversy about whether it is preferable to attempt a radical resection, with wide exposure, extensive dissection, and the potential for significant surgical morbidity, or to determine the oncological features of the tumor and plan local control through more limited approaches. ${ }^{3,11}$ Surgical morbidity can be significant, particularly with standard approaches. Samii et al. reported a neurological complication rate of $33.3 \%$ and an incidence of cerebrospinal fluid leakage of $10.3 \% .^{3,14,15}$ Furthermore, gross-total resection is achievable in only $50 \%$ of cases, and there is no evidence that greater extent of resection improves outcome. ${ }^{3}$ Chondrosarcomas are considered resistant to radiation and chemotherapy. ${ }^{15}$ The use of adjuvant radiation following resection of cranial chondrosarcomas has been shown to improve tumor control and overall survival, but with modest results compared with cases in which patients were treated with surgery alone. ${ }^{3,4,7}$ Almefty et al. mentioned that radiotherapy may not be necessary in patients with low-grade chondrosarcoma. ${ }^{1}$ Due to the location of chondrosarcomas in the skull base, with critical structures located near the tumors, highly conformal radiation techniques, such as proton or carbon ion therapy are required..$^{15}$ Multiple modalities can be used, including fractionated photon radiotherapy, particle radiotherapy, or stereotactic radiosurgery. There are no Class I data suggesting that the use of one radiation modality is preferable to use of any other. ${ }^{3}$ The results of chemotherapy have been discouraging, and therefore it is not part of the standard therapy for patients with this tumor. ${ }^{3,4}$

The current standard for treatment of cranial chondrosarcoma is resection to obtain a definitive tissue diagnosis and reduce the tumor bulk, followed by radiation therapy aimed at preventing tumor recurrence. $3,6,11$ As stated previously, the approach selection is principally determined by the primary direction of tumor growth and the involved cranial nerves. ${ }^{3}$ Bloch and Parsa pointed out that tumors involving the petrous apex and upper third of the clivus, with extension anteriorly into Meckel's cave or the cavernous sinus, should be accessed 


\section{Chondrosarcomas with CPA extension}

through a frontotemporal orbitozygomatic approach or middle-fossa craniotomy with subtemporal dissection. Posteriorly and inferiorly directed tumors going below the internal acoustic canal are usually addressed through a retrosigmoid or transpetrosal approach. Large tumors may require a combined petrosal/middle-fossa approach or a staged procedure. Alternative approaches include transfacial, transbasal, transmaxillary, transoral, transfacial, and transsphenoidal approaches.,11 The majority of open approaches can reach one segment of complex chondrosarcomas, leaving large residual tumors in other compartments. A perfect example is our Case 1, in which experienced neurosurgeons performed a combined presigmoid transpetrous/subtemporal approach resulting in partial tumor resection and significant residual tumor.

The use of EEAs has been reported with success for specific tumors. ${ }^{3,5,16}$ An EEA is generally associated with reduced morbidity because vascular and neural structures are displaced away from the surgical field. ${ }^{6}$ Also, the development of a specific endonasal surgical armamentarium has improved the visibility of the operating field and instrumentation. New techniques for skull base reconstruction, especially vascularized pedicled flaps, ${ }^{10}$ have enabled intradural dissections with acceptable rates of cerebrospinal fluid leaks. ${ }^{911}$ Qiu et al. reported 7 cases of chondrosarcomas in the skull base resected through EEAs, with a median follow-up of 36 months and no recurrence in $71 \% .^{13}$ Zanation et al. achieved gross-total resection of 3 chondrosarcomas of the petrous apex. ${ }^{16}$ Battaglia et al. reported 1 case of gross-total resection of a sinonasal chondrosarcoma through a transpterygoid transmaxillary approach, without complications. ${ }^{2}$ We strongly believe that chondrosarcomas involving the posterior fossa and CPA are amenable to treatment with an EEA approach, with excellent results in terms of resection, while minimizing surgical morbidity, as evidenced by the current series. The main characteristic of the endonasal approach is its ability to reach the petroclival synchondrosis with little to no morbidity for the patient. Once the core of the tumor is accessed, the surgeons can follow the tumor in any direction. With that strategy, tumors are removed from the parapharyngeal area, middle fossa, and posterior fossa. Needless to say, any tumor extension into the sphenoid can be directly accessed through an EEA as well. Neuromonitoring and image guidance are used in all cases, aiding the surgeon to improve resec-

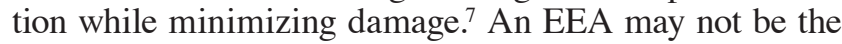
perfect approach for achieving total resection of tumors in these complex cases every single time. However, we believe that an EEA allows better overall tumor exposure and optimized conditions for superior resection. This is particularly true in regard to chondrosarcomas with primary $\mathrm{CPA}$ region involvement, such as the ones in the current study. The alternative to endonasal resection of these lesions would entail a retrosigmoid approach or a variation of a transpetrous approach; these techniques will eventually reach the tumor but will also require incising 2 areas of dura (posterolateral and ventral), traversing a significant portion of disease-free cisternal space, and navigating between the narrow windows provided by the cranial nerves.

Adequate resection has shown a significant association with long-term survival in different studies. ${ }^{4,7}$ Ac- cordingly, we believe in EEA as the main approach for resection of skull base chondrosarcomas, since it enables the surgeon to access multiple compartments without the need for neural retraction. It should be complemented by other approaches in case of initial subtotal resection to achieve an overall gross-total tumor removal before radiation is initiated (as exemplified by Case 4).

\section{Conclusions}

The standard treatment for chondrosarcomas of the skull base is resection, followed by some form of radiation. EEAs offer an alternative for achieving maximal safe resection, even for lesions in the posterior fossa and CPA, providing adequate cytoreduction with minimal morbidity due to surgical manipulation. Other approaches should be used after EEA in cases of subtotal resection to achieve gross-total resection before further treatment is initiated.

\section{Disclosure}

The authors report no conflict of interest concerning the materials or methods used in this study or the findings specified in this paper.

Author contributions to the study and manuscript preparation include the following. Conception and design: Prevedello, Ditzel Filho. Drafting the article: Prevedello, Mesquita Filho, Ditzel Filho, Fiore, Dolci. Critically revising the article: Prevedello, Ditzel Filho. Reviewed submitted version of manuscript: Prevedello, Ditzel Filho, Otto, Carrau. Approved the final version of the manuscript on behalf of all authors: Prevedello. Administrative/technical/material support: Ditzel Filho, Martinez, Dolci. Study supervision: Prevedello, Ditzel Filho.

\section{References}

1. Almefty K, Pravdenkova S, Colli BO, Al-Mefty O, Gokden M: Chordoma and chondrosarcoma: similar, but quite different, skull base tumors. Cancer 110:2457-2467, 2007

2. Battaglia P, Turri-Zanoni M, Dallan I, Gallo S, Sica E, Padoan $\mathrm{G}$, et al: Endoscopic endonasal transpterygoid transmaxillary approach to the infratemporal and upper parapharyngeal tumors. Otolaryngol Head Neck Surg 150:696-702, 2014

3. Bloch O, Parsa AT: Skull base chondrosarcoma: evidence-based treatment paradigms. Neurosurg Clin N Am 24:89-96, 2013

4. Brackmann DE, Teufert KB: Chondrosarcoma of the skull base: long-term follow-up. Otol Neurotol 27:981-991, 2006

5. Frank G, Sciarretta V, Calbucci F, Farneti G, Mazzatenta D, Pasquini E: The endoscopic transnasal transsphenoidal approach for the treatment of cranial base chordomas and chondrosarcomas. Neurosurgery 59 (1 Suppl 1):ONS50-ONS57, 2006

6. Frassanito P, Massimi L, Rigante M, Tamburrini G, Conforti G, Di Rocco C, et al: Recurrent and self-remitting sixth cranial nerve palsy: pathophysiological insight from skull base chondrosarcoma. Report of 2 cases. J Neurosurg Pediatr 12: 633-636, 2013

7. Jones PS, Aghi MK, Muzikansky A, Shih HA, Barker FG II, Curry WT Jr: Outcomes and patterns of care in adult skull base chondrosarcomas from the SEER database. J Clin Neurosci 21:1497-1502, 2014

8. Kassam AB, Gardner P, Snyderman C, Mintz A, Carrau R: Expanded endonasal approach: fully endoscopic, completely transnasal approach to the middle third of the clivus, petrous bone, middle cranial fossa, and infratemporal fossa. Neurosurg Focus 19(1):E6, 2005 


\section{P. M. Mesquita Filho et al.}

9. Kassam AB, Prevedello DM, Carrau RL, Snyderman CH, Thomas A, Gardner P, et al: Endoscopic endonasal skull base surgery: analysis of complications in the authors' initial 800 patients. A review. J Neurosurg 114:1544-1568, 2011

10. Kassam AB, Thomas A, Carrau RL, Snyderman CH, Vescan A, Prevedello D, et al: Endoscopic reconstruction of the cranial base using a pedicled nasoseptal flap. Neurosurgery 63 (1 Suppl 1):ONS44-ONS53, 2008

11. Kuge A, Sato S, Sakurada K, Takemura S, Kikuchi Z, Saito Y, et al: Technical notes on endoscopic transnasal transsphenoidal approach for clival chondrosarcoma. Sarcoma 2011: 953047, 2011

12. Pickett BP, Hepworth EJ: Neoplasms of the ear and lateral skull base, in Bailey BJ, Calhoun KH, Healy GB, et al (eds): Head and Neck Surgery-Otolaryngology, ed 3. Philadelphia: Lippincott Williams \& Wilkins, 2001, pp 1725-1744

13. Qiu QH, Liang MZ, Liu H, Chen SH, Zhang HB, Zhang QH: [Nasal endoscopic surgical treatment for chondrosarcoma of paranasal sinus and the skull base.] Zhonghua Er Bi Yan Hou Tou Jing Wai Ke Za Zhi 45:551-554, 2010 (Chinese)
14. Samii A, Gerganov V, Herold C, Gharabaghi A, Hayashi N, Samii M: Surgical treatment of skull base chondrosarcomas. Neurosurg Rev 32:67-75, 2009

15. Uhl M, Mattke M, Welzel T, Oelmann J, Habl G, Jensen AD, et al: High control rate in patients with chondrosarcoma of the skull base after carbon ion therapy: first report of long-term results. Cancer 120:1579-1585, 2014

16. Zanation AM, Snyderman CH, Carrau RL, Gardner PA, Prevedello DM, Kassam AB: Endoscopic endonasal surgery for petrous apex lesions. Laryngoscope 119:19-25, 2009

Manuscript submitted June 15, 2014.

Accepted July 24, 2014.

Please include this information when citing this paper: DOI: 10.3171/2014.7.FOCUS 14349.

Address correspondence to: Daniel M. Prevedello, M.D., Department of Neurological Surgery, The Ohio State University Wexner Medical Center, 410 W. 10th Ave., N-1049 Doan Hall, Columbus, OH 43210. email: daniel.prevedello@osumc.edu. 